\title{
Correction to: Deep kernel learning in extreme learning machines
}

\author{
A. L. Afzal ${ }^{1}$ (D) Nikhitha K. Nair ${ }^{2} \cdot$ S. Asharaf ${ }^{2}$
}

Published online: 2 January 2021

(c) Springer-Verlag London Ltd., part of Springer Nature 2020

\section{Correction to: Pattern Analysis and Applications https://doi.org/10.1007/s10044-020-00891-8}

In the original publication of the article, the Acknowledgements section was missed unfortunately. The correct Acknowledgements section is given in this correction.

Acknowledgements This work has been supported by Kerala State Council for Science, Technology and Environment (KSCSTE) under the fellowship No. 48/FSHP/2016/ KSCSTE.

The original article has been updated.

Publisher's Note Springer Nature remains neutral with regard to jurisdictional claims in published maps and institutional affiliations.

The original article can be found online at https://doi.org/10.1007/ s10044-020-00891-8.

A. L. Afzal

afzal.res15@iiitmk.ac.in

Nikhitha K. Nair

nikhitha.res17@iiitmk.ac.in

S. Asharaf

asharaf.s@iiitmk.ac.in

1 College of Engineering Muttathara, Thiruvananthapuram, India

2 Data Engineering Lab, Indian Institute of Information Technology and Management-Kerala (IIITM-K),

Thiruvananthapuram, India 